\title{
Ferromagnetic behaviour in the strongly interacting two-component Bose gas
}

\author{
Xi-Wen Guan ${ }^{1}$, Murray T. Batchelor ${ }^{1,2}$ and Minoru Takahashi ${ }^{3}$ \\ ${ }^{1}$ Department of Theoretical Physics, Research School of Physical Sciences and Engineering, \\ Australian National University, Canberra ACT 0200, Australia \\ ${ }^{2}$ Mathematical Sciences Institute, Australian National University, Canberra ACT 0200, Australia \\ ${ }^{3}$ Department of Physics, Toho University, Miyama 2-2-1, Funabashi 274-8510, Japan
}

(Dated: August 17, 2021)

\begin{abstract}
We investigate the low temperature behaviour of the integrable $1 \mathrm{D}$ two-component spinor Bose gas using the thermodynamic Bethe ansatz. We find that for strong coupling the characteristics of the thermodynamics at low temperatures are quantitatively affected by the spin ferromagnetic states, which are described by an effective ferromagnetic Heisenberg chain. The free energy, specific heat, susceptibility and local pair correlation function are calculated for various physical regimes in terms of temperature and interaction strength. These thermodynamic properties reveal spin effects which are significantly different than those of the spinless Bose gas. The zero-field susceptibility for finite strong repulsion exceeds that of a free spin paramagnet. The critical exponents of the specific heat $c_{v} \sim T^{1 / 2}$ and the susceptibility $\chi \sim T^{-2}$ are indicative of the ferromagnetic signature of the two-component spinor Bose gas. Our analytic results are consistent with general arguments by Eisenberg and Lieb for polarized spinor bosons.
\end{abstract}

PACS numbers: 03.75.Hh, 03.75.Mn, 04.20.Jb, 05.30.Jp

\section{INTRODUCTION}

Experiments with ultracold quantum gases are opening up exciting new possibilities for testing and exploring quantum effects in many-body systems (for recent reviews, see Refs. [1-3]). These include experiments on effectively one-dimensional (1D) quantum Bose gases of ${ }^{87} \mathrm{Rb}$ atoms in which the interaction strength between atoms is tunable [4-8]. The experiments provide a striking example of realizing an integrable quantum many-body problem. They demonstrate the explicit fermionization of bosons and provide a direct test of theoretical results obtained for the integrable 1D interacting (spinless) Bose gas $[9,10]$. Another frontier of activity involves spinor Bose gases of alkali atoms in which hyperfine states comprise the pseudospins [11-13]. In these systems quantum collisional effects can produce spatio-temporal spin oscillations (spin waves) [14-17]. The observation of collective dynamics of spin waves and spin-state segregation in trapped spinor Bose gases has stimulated a wide range of interest in studying magnetism, topological spin defects and novel quantum phase transitions in spinor Bose gases [18, 19].

Two-component spinor Bose gases have been experimentally created in a magnetic trap by rotating two hyperfine states so that the two atomic hyperfine states make up a pseudo-spin doublet [20], e.g., the $\left|F=2, m_{F}=-1\right\rangle$ and $\left|F=1, m_{F}=1\right\rangle$ hyperfine states of ${ }^{87} \mathrm{Rb}$. In general, spin-independent s-wave scattering dominates interactions in alkali atomic gases. In 1D, the two-component Bose gas with spin-independent s-wave scattering can be exactly solved, like the spinless model, by means of the Bethe ansatz [21, 22]. In contrast to Fermi gases, ferromagnetic order emerges in spinor Bose gases as long as the interaction is fully spin independent $[15,23]$. The low-energy excitations of the model split into collective excitations carrying charge and collective excitations carrying spin. The charge excitations are phonons whereas the spin excitations have quadratic dispersion connected to spin wave excitations $[24,25]$. Spin dynamics in the 1D ferromagnetic Bose gas have been studied recently [26]. Girardeau's Fermi-Bose mapping has been used to study the 1D spinor Bose gases [27]. In general the two-component interacting Bose gas provides a tunable testing ground for observing the phenomenon of spin-charge separation [28].

Quantum gases with multi-spin states are expected to exhibit even richer quantum effects than their single component counterparts [1-3]. Universal features appearing in the low temperature behaviour of strongly interacting spinor Bose gases should differ significantly from those of spinless Bose gases and the antiferromagnetic behaviour of Fermi gases due to their fundamentally different statistical signatures. One way to calculate the thermodynamics of integrable many-body systems is via the thermodynamic Bethe ansatz (TBA) [29-33], introduced by Yang and Yang [34] for the 1D Bose gas. However, it is a challenging problem to derive exact TBA results for the thermodynamics of 1D quantum many-body systems. Our aim here is to obtain universal characteristics of ferromagnetic behaviour for the 1D two-component strongly interacting Bose gas of ultracold atoms via the TBA method. We will see that the ferromagnetic phase associated with the spin degrees of freedom may separate from the gas phase in the strongly repulsive regime due to spin-charge separation. The low temperature behaviour is dominated by the spin ferromagnetic states, which are described by an effective ferromagnetic Heisenberg chain. In this way we make contact with the known results for the thermodynamics of the ferromagnetic Heisenberg chain $[35,36]$ to derive analytic expressions for the free energy, specific heat, susceptibility and local pair correlation function for the strongly interacting two-component 
Bose gas in terms of temperature and interaction strength. These thermodynamic properties reveal some novel spin effects. Our explicit results are consistent with general arguments by Eisenberg and Lieb [23] for polarized spinor bosons.

This paper is set out as follows. In section II we present the Bethe ansatz solution of the 1D two-component interacting Bose gas. The ground state properties are also calculated. In section III we introduce the TBA for the spinor Bose gas in order to study the thermodynamics at low temperatures, including the analysis of spin charge separation. The ferromagnetic ground state is studied by means of the solution of the TBA equations in section IV. We discuss low temperature ferromagnetic behaviour for the 1D strongly interacting Bose gas of atoms in section V. The local pair correlation function is studied at low temperatures in section VI. Section VII is devoted to a brief summary and concluding remarks.

\section{THE TWO-COMPONENT SPINOR BOSE GAS}

The Hamiltonian describing a $\delta$-function interacting gas of $N$ bosons of mass $m$ constrained by periodic boundary conditions to a line of length $L$ with internal degrees of freedom is

$$
\mathcal{H}=-\frac{\hbar^{2}}{2 m} \sum_{i=1}^{N} \frac{\partial^{2}}{\partial x_{i}^{2}}+g_{1 \mathrm{D}} \sum_{1 \leq i<j \leq N} \delta\left(x_{i}-x_{j}\right) .
$$

For the ultracold atomic gases [37], the coupling constant $g_{1 \mathrm{D}}$ can be written in terms of the scattering strength $c=2 / a_{1 \mathrm{D}}$ as $g_{1 \mathrm{D}}=\hbar^{2} c / m$. The effective $1 \mathrm{D}$ scattering length $a_{1 \mathrm{D}}$ can be related to the $3 \mathrm{D}$ scattering length for bosons or fermions confined in a 1D geometry. The dimensionless coupling constant $\gamma=c / n=m g_{1 \mathrm{D}} /\left(\hbar^{2} n\right)$ is convenient for physical analysis. Here $n=N / L$ is the linear density. We take $2 m=\hbar=1$ for simplicity in the following equations. However, we reinstate them where appropriate in discussing the thermodynamics of the model. The wavefunctions of Hamiltonian (1) for the spinor Bose gas are symmetric under exchange of spatial and internal spin coordinates between two particles. We shall see that this statistical signature triggers rather novel ferromagnetic behaviour in the degenerate quantum spinor Bose gas. The interaction is attractive for $g_{1 \mathrm{D}}<0$ and repulsive for $g_{1 \mathrm{D}}>0$. However, one should note that there is no thermodynamic limit for the attractive case in Bose gases.

For $M$ spin-down bosons, the Bethe ansatz equations (BAE) for the two-component Bose gas are of the form [21, 22]

$$
\begin{aligned}
\exp \left(\mathrm{i} k_{j} L\right) & =-\prod_{\ell=1}^{N} \frac{k_{j}-k_{\ell}+\mathrm{i} c}{k_{j}-k_{\ell}-\mathrm{i} c} \prod_{\alpha=1}^{M} \frac{k_{j}-\lambda_{\alpha}-\frac{1}{2} \mathrm{i} c}{k_{j}-\lambda_{\alpha}+\frac{1}{2} \mathrm{i} c}, \quad j=1, \ldots, N \\
\prod_{\ell=1}^{N} \frac{\lambda_{\alpha}-k_{\ell}-\frac{1}{2} \mathrm{i} c}{\lambda_{\alpha}-k_{\ell}+\frac{1}{2} \mathrm{i} c} & =-\prod_{\beta=1}^{M} \frac{\lambda_{\alpha}-\lambda_{\beta}-\mathrm{i} c}{\lambda_{\alpha}-\lambda_{\beta}+\mathrm{i} c}, \quad \alpha=1, \ldots, M
\end{aligned}
$$

in terms of which the energy eigenspectrum is given by $E=\sum_{j=1}^{N} k_{j}^{2}$. In the thermodynamic limit, i.e., $N, L \rightarrow \infty$ with $N / L$ finite, these equations can be written as coupled integral equations in terms of the particle and hole root densities $\rho(k)$ and $\rho^{h}(k)\left(\sigma(\lambda)\right.$ and $\left.\sigma^{h}(\lambda)\right)$ for the charge (spin) degrees of freedom, respectively. These are

$$
\begin{aligned}
\rho(k)+\rho^{h}(k) & =\frac{1}{2 \pi}+\frac{1}{2 \pi} \int_{-Q}^{Q} \frac{2 c \rho\left(k^{\prime}\right)}{c^{2}+\left(k-k^{\prime}\right)^{2}} d k^{\prime}-\frac{1}{2 \pi} \int_{-B}^{B} \frac{c \sigma(\lambda)}{c^{2} / 4+(k-\lambda)^{2}} d \lambda \\
\sigma(\lambda)+\sigma^{h}(\lambda) & =\frac{1}{2 \pi} \int_{-Q}^{Q} \frac{c \rho(k)}{c^{2} / 4+(\lambda-k)^{2}} d k-\frac{1}{2 \pi} \int_{-B}^{B} \frac{2 c \sigma(\lambda)}{c^{2}+\left(\lambda-\lambda^{\prime}\right)^{2}} d \lambda^{\prime} .
\end{aligned}
$$

The integration limits $Q$ and $B$ are determined by $N / L=\int_{-Q}^{Q} \rho(k) d k$ and $M / L=\int_{-B}^{B} \sigma(\lambda) d \lambda$. At zero temperature, the ground state corresponds to the configuration $\sigma(\lambda)=\rho^{h}(k)=0$ leading to a ferromagnetic ground state. For the ground state there are therefore no holes in the charge degrees of freedom and no quasiparticles in the spin degrees of freedom. However, as the temperature increases spin strings become involved in the thermal equilibrium states. We shall investigate this ground state configuration via analysis of the string solutions to the TBA.

\section{THE THERMODYNAMIC BETHE ANSATZ}

For finite temperatures each of the $N$ quasimomenta $k_{i}$ are real due to the repulsive interaction. However, the spin quasimomenta form complex strings of the form [38] $\lambda_{\alpha, j}^{n}=\Lambda_{\alpha}^{n}+\mathrm{i}(n+1-2 j) c / 2$ for $j=1, \ldots, n$. Here the number 
of strings $\alpha=1, \ldots, N_{n} . \Lambda_{\alpha}^{n}$ on the real axis denotes the position of the centre of a length- $n$ string. The number of $n$-strings $N_{n}$ satisfies the relation $M=\sum_{n} n N_{n}$. It is assumed that the distribution of Bethe roots along the real axis is dense enough to pass to the continuum limit. After performing a standard calculation with the string solutions and introducing the convolution integral $(f * g)(\lambda)=\int_{-\infty}^{\infty} f\left(\lambda-\lambda^{\prime}\right) g\left(\lambda^{\prime}\right) d \lambda^{\prime}$, the BAE (2) become

$$
\begin{aligned}
\rho(k)+\rho^{h}(k) & =\frac{1}{2 \pi}+\frac{1}{2 \pi} \int_{-\infty}^{\infty} \frac{2 c \rho\left(k^{\prime}\right) d k^{\prime}}{c^{2}+\left(k-k^{\prime}\right)^{2}}-\sum_{n=1}^{\infty} a_{n} * \sigma_{n}(k) \\
\sigma_{n}(\lambda)+\sigma_{n}^{h}(\lambda) & =a_{n} * \rho(\lambda)-\sum_{m=1}^{\infty} T_{n m} * \sigma_{m}(\lambda)
\end{aligned}
$$

where

$$
T_{n m}(\lambda)=\left\{\begin{array}{l}
a_{|n-m|}(\lambda)+2 a_{|n-m|+2}(\lambda)+\ldots+2 a_{n+m-2}(\lambda)+a_{n+m}(\lambda), \text { for } n \neq m \\
2 a_{2}(\lambda)+2 a_{4}(\lambda)+\ldots+2 a_{2 n-2}(\lambda)+a_{2 n}(\lambda), \text { for } n=m
\end{array}\right.
$$

and

$$
a_{n}(\lambda)=\frac{1}{2 \pi} \frac{n c}{(n c / 2)^{2}+\lambda^{2}} .
$$

The equilibrium states at finite temperature $T$ are described by the equilibrium particle and hole densities $\rho(k)$ and $\rho^{h}(k)$ of the charge degrees of freedom and the equilibrium string densities $\sigma_{n}(\lambda)$ and $\sigma_{n}^{h}(\lambda)$ of the spin degrees of freedom. Here $n=1,2, \ldots, \infty$. The partition function $Z=\operatorname{tr}\left(\mathrm{e}^{-\mathcal{H} / T}\right)$ is defined by

$$
Z=\sum_{\rho, \rho^{h}, \sigma_{n}, \sigma_{n}^{h}} W\left(\rho, \rho^{h}, \sigma_{n}, \sigma_{n}^{h}\right) \mathrm{e}^{-E\left(\rho, \rho^{h}, \sigma_{n}, \sigma_{n}^{h}\right) / T}
$$

where the densities satisfy (4) with $W\left(\rho, \rho^{h}, \sigma_{n}, \sigma_{n}^{h}\right)$ the number of states corresponding to the given densities. Introducing the combinatorial entropy $S=\ln W\left(\rho, \rho^{h}, \sigma_{n}, \sigma_{n}^{h}\right)$, the grand partition function is $Z=\mathrm{e}^{-G / T}$, where the Gibbs free energy $G=E-\mu N-H\left(N_{\uparrow}-N_{\downarrow}\right) / 2-T S$. Here $\mu$ is the chemical potential and $N_{\uparrow}\left(N_{\downarrow}\right)$ denotes the number of the particles with up (down) spin. Recall that $N_{\downarrow}=M$. The energy per unit length is defined by

$$
E / L=\int_{-\infty}^{\infty} k^{2} \rho(k) d k-m^{z} H .
$$

Here $H$ is the external magnetic field and $m^{z}=\left(N_{\uparrow}-N_{\downarrow}\right) / 2$ denotes the atomic magnetic momentum (where the Bohr magneton $\mu_{B}$ and the Lande factore are absorbed into the magnetic field $\left.H\right)$. The magnetization per unit length in the $z$-direction is thus given by

$$
m^{z}=\frac{1}{2} \int_{-\infty}^{\infty} \rho(k) d k-\sum_{n} n \int_{-\infty}^{\infty} \sigma_{n}(\lambda) \mathrm{d} \lambda
$$

Now the equilibrium states are determined by the minimization condition of the Gibbs free energy [30, 34], i.e., the condition $\delta(E-\mu n-T S)=0$, which gives rise to the set of coupled nonlinear integral equations (the TBA equations) $[39]$

$$
\begin{aligned}
\epsilon(k) & =k^{2}-\mu-\frac{1}{2} H-\frac{T}{2 \pi} \int_{-\infty}^{\infty} \frac{2 c}{c^{2}+\left(k-k^{\prime}\right)^{2}} \ln \left(1+\mathrm{e}^{-\epsilon\left(k^{\prime}\right) / T}\right)-T \sum_{n=1}^{\infty} a_{n}(k-\lambda) * \ln \left(1+\eta_{n}^{-1}(\lambda)\right) \\
\ln \eta_{n}(\lambda) & =\frac{n H}{T}+a_{n}(\lambda-k) * \ln \left(1+\mathrm{e}^{-\epsilon\left(k^{\prime}\right) / T}\right)+\sum_{n=1}^{\infty} T_{m n}\left(\lambda-\lambda^{\prime}\right) * \ln \left(1+\eta_{n}^{-1}\left(\lambda^{\prime}\right)\right) .
\end{aligned}
$$

Here we have defined the dressed energy $\epsilon(k):=T \ln \left(\rho^{h}(k) / \rho(k)\right)$ with respect to the quasimomentum $k$. Similarly $\eta(\lambda):=\sigma^{h}(\lambda) / \sigma(\lambda)$ with respect to the spin quasimomentum $\lambda$. The dressed energy $\epsilon(k)$ plays the role of excitation energy measured from the Fermi energy level $\epsilon\left(k_{\mathrm{F}}\right)=0$, where $k_{\mathrm{F}}$ is the Fermi momentum. The pressure $P(T, H)$ and free energy $F(T, H)$ per unit length in the thermodynamic limit are given in terms of the dressed energy by

$$
\begin{aligned}
& P(T, H)=\frac{T}{2 \pi} \int_{-\infty}^{\infty} d k \ln \left(1+\mathrm{e}^{-\epsilon(k) / T}\right) \\
& F(T, H)=\mu n-\frac{T}{2 \pi} \int_{-\infty}^{\infty} d k \ln \left(1+\mathrm{e}^{-\epsilon(k) / T}\right) .
\end{aligned}
$$


At low temperatures and zero magnetic field, it is important to note that $\mathrm{e}^{-\epsilon(k) / T}$ is negligibly small if the energy $\frac{\hbar^{2} k^{2}}{2 m}>2 \mu$, where $\mu$ is the chemical potential. It follows that for strong coupling, i.e., for $\gamma \gg 1$, the TBA equations (10) can be written as

$$
\begin{aligned}
\epsilon(k) & =k^{2}-\mu-\frac{1}{2} H-\frac{2 c P(T, H)}{c^{2}+k^{2}}-T \sum_{n=1}^{\infty} \int_{-\infty}^{\infty} a_{n}(\lambda) \ln \left(1+\eta_{n}^{-1}(\lambda)\right) \\
\ln \eta_{n}(\lambda) & =\frac{n H}{T}+\frac{4 \pi P(T, H) a_{n}(\lambda)}{c T}+\sum_{n=1}^{\infty} T_{m n}\left(\lambda-\lambda^{\prime}\right) * \ln \left(1+\eta_{n}^{-1}\left(\lambda^{\prime}\right)\right) .
\end{aligned}
$$

In the above equations, we have only kept terms to order $1 / c$ for the dressed enery $\epsilon(k)$. We have also made the variable changes $\lambda \rightarrow c \lambda / 2$ and $\eta(\lambda) \rightarrow \eta(c \lambda / 2)$. Thus the function $a_{n}(\lambda)$ defined in (6) becomes $a_{n}(x)=\frac{1}{\pi} \frac{n}{n^{2}+x^{2}}$, which is Takahashi's notation for the ferromagnetic Heisenberg chain [30]. It will be clearly seen from the TBA equations (13) that the low temperature behaviour of the strongly interacting two-component boson model is determined by the hard core Bose gas state and ferromagnetic spin wave fluctuations described by the ferromagnetic Heisenberg chain with an effective coupling strength $J=2 P(T, H) / c>0$. We shall see through the TBA equations (13) that the temperature dependent part of this effective coupling strength contributes to the free energy at $O\left(T^{3}\right)$. At low temperatures $J \rightarrow 4 E_{\mathrm{F}} / 3$, where $E_{\mathrm{F}}$ is the Fermi energy.

Following the analysis of the TBA equations for the ferromagnetic Heisenberg chain [30], the TBA equations (13) can be rewritten in terms of the function $\eta_{1}(\lambda)$ in the form

$$
\epsilon(k) \approx k^{2}-\mu-\frac{2 c P(T, H)}{c^{2}+k^{2}}+f_{X X X}(T, H)
$$

where

$$
f_{X X X}(T, H) \approx J \ln 2-T \int_{-\infty}^{\infty} d \lambda s(\lambda) \ln \left(1+\eta_{1}(\lambda)\right)
$$

is the free energy of a ferromagnetic Heisenberg chain with coupling $J \approx 2 P(T, H) / c$. Here $1 / s(\lambda)=4 \cosh (\pi \lambda / 2)$. The function $\eta_{1}(\lambda)$ is determined by the TBA equations for the ferromagnetic Heisenberg chain [30], which are

$$
\begin{aligned}
\ln \eta_{1}(\lambda) & =\frac{2 \pi J}{T} s(\lambda)+s * \ln \left(1+\eta_{2}(\lambda)\right) \\
\ln \eta_{n}(\lambda) & =s * \ln \left(1+\eta_{n-1}(\lambda)\right) \ln \left(1+\eta_{n+1}(\lambda)\right) .
\end{aligned}
$$

For large $n$, the string solutions obey $\lim _{n \rightarrow \infty} n^{-1} \ln \eta_{n}(\lambda)=H / T$.

So far we have separated the ferromagnetic spin state from the hard core gas phase via the TBA formalism. It is important to note that for the strong coupling regime the spin and charge degrees of freedom are coupled via the pressure. The spin velocity vanishes whereas the charge velocity tends to the Fermi velocity of noninteracting spinless fermions as $\gamma \rightarrow \infty$. In this extreme case, the effective mass takes the maximum value $m^{*}=N m$, meaning that by moving one boson with down spin, one has to move all the particles with up spins [24, 25]. For finitely strong repulsion, a quadratic dispersion of spin wave excitations above the ferromagnetic ground state can be obtained from the Bethe ansatz solution (2) $[24,25]$. In general spin charge separation is a typical phenomenon in interacting many-body systems [28].

Before moving on to discuss the solutions of the TBA (16), we proceed to calculate the pressure (11). Without loss of generality, we let $\epsilon(k)=\frac{\hbar^{2} k^{2}}{2 m}-A(T, H)$, where $A(T, H)=\mu+2 P(T, H) / c-f_{X X X}(T, H)$. Hereafter we restore physical units in the thermodynamic properties. Integration by parts gives

$$
P(T, H) \approx \frac{1}{\sqrt{\frac{\pi^{2} \hbar^{2}}{2 m}}} \int_{0}^{\infty} \frac{\sqrt{\epsilon} d \epsilon}{1+\mathrm{e}^{\frac{\epsilon-A(T, H)}{K_{B} T}}} .
$$

The integral in (17) can be calculated explicitly using Sommerfeld expansion [40] to give

$$
P(T, H) \approx \frac{1}{\sqrt{\frac{\pi^{2} \hbar^{2}}{2 m}}} \frac{2}{3} A(T, H)^{\frac{3}{2}}\left[1+\frac{\pi^{2}}{8}\left(\frac{K_{B} T}{A(T, H)}\right)^{2}+\frac{7 \pi^{4}}{640}\left(\frac{K_{B} T}{A(T, H)}\right)^{4}+\cdots\right] .
$$

Here the function $A(T, H)$ contains $P(T, H)$. From equation (18) we can find a relation between the pressure $P(T, H)$ and chemical potential $\mu$ by iteration. This provides a starting point to calculate the thermodynamics of the strongly interacting two-component Bose gas at low temperatures, including the zero temperature limit. 


\section{THE GROUND STATE AND THE SPINLESS BOSE GAS}

Pure dynamical interaction drives the spinless Bose gas into distinct quantum phases of matter: from the quasiBose-Einstein condensate to the Tonks-Girardeau phase. As stated in the introduction, this elegantly simple Bethe ansatz solved 1D quantum many-body system $[9,10]$ is testable in experiments on trapped quantum gases of ultracold atoms [4-7]. It is natural to expect that spin dynamics in the interacting two-component spinor Bose gases would lead to significantly different quantum effects than those of the spinless Bose gas. At $T \rightarrow 0$, it is suitable to use the dressed energy formalism in the TBA equations (16), i.e.,

$$
\begin{aligned}
& \xi_{1}(\lambda)=2 \pi J s(\lambda)+s * \xi_{2}^{+}(\lambda) \\
& \xi_{n}(\lambda)=s *\left(\xi_{n-1}^{+}(\lambda)+\xi_{n+1}^{+}(\lambda)\right)
\end{aligned}
$$

where $\lim _{n \rightarrow \infty} \xi_{n} / n=H$. Here the dressed energy is defined as $\xi_{n}(\lambda):=T \ln \eta_{n}(\lambda)$, with $\xi_{n}^{+}(\lambda)\left(\xi_{n}^{-}(\lambda)\right)$ denoting the dressed energy for $\xi_{n}(\lambda) \geq 0\left(\xi_{n}(\lambda)<0\right)$. The free energy is now

$$
\begin{aligned}
f_{X X X}(T, H) & =-\frac{1}{2} H+\sum_{n=1}^{\infty} \int_{-\infty}^{\infty} a_{n}(\lambda) \xi_{n}^{-}(\lambda) d \lambda \\
& =J \ln 2-\int_{-\infty}^{\infty} d \lambda s(\lambda) \xi_{1}^{+}(\lambda) .
\end{aligned}
$$

For the ferromagnetic case $J>0$, the solution of the TBA (19) is given by [30]

$$
\xi_{n}(\lambda)=\xi_{n}^{+}(\lambda)=2 \pi J a_{n}(\lambda)+H n
$$

where $n=1,2, \ldots, \infty$. Here we see that for $T=0, f_{X X X}=-H / 2$. Therefore it follows that the fully-polarized state forms a ferromagnetic ground state. In this way the TBA gives rise to a direct proof of the existence of the ferromagnetic ground state.

In order to understand spin effects in the spinor Bose gas, we first discuss the low temperature behaviour of the spinless Bose gas. It was for this model that Yang and Yang introduced the TBA formalism, with result

$$
\epsilon(k)=\epsilon^{0}(k)-\mu-\frac{T}{2 \pi} \int_{\infty}^{\infty} d k^{\prime} \frac{2 c}{c^{2}+\left(k-k^{\prime}\right)^{2}} \ln \left(1+\mathrm{e}^{-\epsilon\left(k^{\prime}\right) / T}\right)
$$

which is the special case of the TBA equation (10) for the spinor Bose gas. At zero temperature, one can obtain physical quantities, such as the ground state energy per unit length $E_{0}$, chemical potential $\mu$, pressure $P_{0}$ and the cut-off momentum $Q$. In the strong coupling limit the results for these quantities are

$$
\begin{aligned}
& E_{0} \approx \frac{1}{3} n^{3} \pi^{2}\left(1-\frac{4}{\gamma}\right), \quad \mu_{0} \approx n^{2} \pi^{2}\left(1-\frac{16}{3 \gamma}\right), \\
& P_{0} \approx \frac{2}{3} n^{3} \pi^{2}\left(1-\frac{6}{\gamma}\right), \quad Q \approx n \pi\left(1-\frac{2}{\gamma}\right) .
\end{aligned}
$$

The macroscopic velocity is

$$
v_{c}=\sqrt{2 \frac{\partial P_{0}}{\partial n}} \approx \frac{\hbar \pi n}{m} \pi\left(1-\frac{4}{\gamma}\right)
$$

The low temperature thermodynamics can also be calculated directly from the pressure (18). For the spinless case, the function $A(T, 0) \approx \mu\left(1+\frac{2 P(T, 0)}{c \mu}\right)$. Substituting $A(T, 0)$ into equation (18) and using the relation $\partial P(T, 0) / \partial \mu=n$ gives the chemical potential

$$
\mu \approx \mu_{0}\left[1+\frac{\pi^{2}}{12}\left(1-\frac{16}{3 \gamma}\right)\left(\frac{K_{B} T}{\mu_{0}}\right)^{2}+\frac{\pi^{4}}{36}\left(1-\frac{32}{5 \gamma}\right)\left(\frac{K_{B} T}{\mu_{0}}\right)^{4}\right]
$$

Here $\mu_{0} \approx n^{2} \pi^{2}\left(1-\frac{16}{3 \gamma}\right)$ coincides with the result given in equation (23). In terms of the degenerate temperature $\tau=K_{B} T / T_{d}$ the free energy per unit length follows from relation (12) as

$$
F(\tau) \approx E_{0}\left[1-\frac{\tau^{2}}{4 \pi^{2}}\left(1+\frac{8}{\gamma}\right)-\frac{\tau^{4}}{60 \pi^{4}}\left(1+\frac{16}{\gamma}\right)\right]
$$


The ground state energy $E_{0}$ agrees with the result given in (23). The expression (18) indeed provides a simple way to derive the thermodynamics. In addition the results (25) and (26) obtained for the spinless Bose gas via the TBA are in good agreement with results derived from generalized exclusion statistics [41]. The results (25) and (26) characterize the low temperature behaviour of the 1D spinless Bose gas induced by the dynamical interaction in strong coupling regime. The specific heat $c_{v}$ and the entropy $S$ follow from the free energy (26) as

$$
\begin{gathered}
c_{v}=-\frac{T L \partial^{2} F(T, 0)}{\partial T^{2}} \approx \frac{N K_{B} \tau}{6\left(1-\frac{4}{\gamma}\right)}+\frac{N K_{B} \tau^{3}}{15 \pi^{2}\left(1-\frac{12}{\gamma}\right)} \\
S=-\frac{T L \partial F(T, 0)}{\partial T} \approx \frac{N K_{B} \tau}{6\left(1-\frac{4}{\gamma}\right)}+\frac{N K_{B} \tau^{3}}{45 \pi^{2}\left(1-\frac{12}{\gamma}\right)}
\end{gathered}
$$

which coincide with the results given in Refs. [41, 44]. Here the degenerate temperature $\tau=K_{B} T / T_{d}$, with $T_{d}=\frac{\hbar^{2} n^{2}}{2 m}$. The total energy per unit length follows from the relation $E(T, 0)=F(T, 0)+S T$, with result

$$
E(\tau) \approx E_{0}\left[1+\frac{\tau^{2}}{4 \pi^{2}}\left(1+\frac{8}{\gamma}\right)+\frac{\tau^{4}}{20 \pi^{4}}\left(1+\frac{16}{\gamma}\right)\right]
$$

The ground state energy $E_{0}$ is as given in (23). From (26) we see that

$$
F(T)=F(0)-\frac{\pi C\left(K_{B} T\right)^{2}}{6 \hbar v_{c}}+O\left(T^{2}\right) .
$$

as expected from conformal field theory arguments for a critical system, i.e., for a system with massless excitations [42] Here the central charge $C=1$ and $v_{c}$ is given by (24). Similarly the finite-size corrections [43] are given by

$$
E(L, N)-L e_{\infty}=-\frac{\hbar \pi C v_{c}}{6 L}+O\left(1 / L^{2}\right)
$$

Here $E(L, N)$ is the finite size ground state energy and $e_{\infty}$ is the energy per unit length in the thermodynamic limit.

Furthermore, at low temperatures the strongly interacting spinless 1D Bose gas can be viewed as a system of ideal particles obeying nonmutual generalized exclusion statistics (GES) with statistics parameter [41, 45] $\alpha \approx 1-2 / \gamma$. These particles obey GES interpolating between bosons and fermions [46-48]. For 1D interacting many-body systems the pairwise dynamical interaction between identical particles is inextricably related to their statistical interaction through scattering. GES is thus the result of collective behaviour exhibited in 1D quantum many body systems. From the GES approach, the free energy and the total energy per unit length are given by [41]

$$
\begin{aligned}
& F(\tau) \approx E_{0}\left[1-\frac{\tau^{2}}{4 \pi^{2}}\left(1+\frac{2}{\gamma}\right)+\frac{3 \xi(3) \tau^{3}}{2 \gamma \pi^{6}}\left(1+\frac{4}{\gamma}\right)-\frac{\tau^{4}}{60 \pi^{4}}\left(1+\frac{4}{\gamma}\right)\right] \\
& E(\tau) \approx E_{0}\left[1+\frac{\tau^{2}}{4 \pi^{2}}\left(1+\frac{2}{\gamma}\right)-\frac{3 \xi(3) \tau^{3}}{\gamma \pi^{6}}\left(1+\frac{4}{\gamma}\right)+\frac{\tau^{4}}{20 \pi^{4}}\left(1+\frac{4}{\gamma}\right)\right] .
\end{aligned}
$$

Here $\zeta(3)=\sum_{n=1}^{\infty} 1 / n^{3}$.

\section{LOW TEMPERATURE FERROMAGNETIC BEHAVIOUR}

The low temperature behaviour of the spinor Bose gas, triggered by the ferromagnetic spin-spin interaction, is intimately related to the thermodynamic behaviour of the ferromagnetic Heisenberg chain, which has been extensively studied via various methods, e.g., numerics [49, 50], spin wave theory [36] and the TBA approach with extrapolation [51]. Although there has been a wide range of interest in the ferromagnetic Heisenberg chain, realization of ferromagnet chains are relatively rare [52]. Most recent interest in the ferromagnetic Heisenberg chain has been from the perspective of string theory $[53,54]$. Obtaining exact analytical results for the thermodynamics of this model still provides a number of open challenges. For one, it is extremely hard to solve the infinitely many equations (16) involved in the TBA. Nevertheless, Takahashi and his coworkers $[36,51]$ have given some results for the free energy and susceptibility which are generally accepted. Schlottmann [35] has also predicted the leading order of the specific heat and zero-field susceptibility via analysis of the string solutions to the TBA equations (16). With the help of these known results for the ferromagnetic Heisenberg chain, we show here that, in the strong coupling regime, the ferromagnetic state induced by the internal spin-spin interaction significantly affects the low temperature behaviour of the two-component spinor Bose gas. 


\section{A. Paramagnet: $\gamma \rightarrow \infty$}

We first consider the extreme case $\gamma \rightarrow \infty$, or say $\gamma \gg 1 / K_{B} T$. In this case the driving term in the TBA equations (16) vanishes as $\gamma \rightarrow \infty$. Thus the string solutions are given by [30]

$$
\eta_{n}(\lambda) \approx\left[\frac{\sinh \left(\frac{(n+1) H}{2 T}\right)}{\sinh \left(\frac{H}{2 T}\right)}\right]^{2}-1
$$

which are known as free spin solutions. In this case, the particles with down-spins are unable to exchange their positions with the particles with up-spins. The spins are thus frozen locally and the spin-spin exchange interaction vanishes. In this case, the statistical interaction is completely suppressed due to the strong repulsion. In this sense, for $\gamma \rightarrow \infty$ both the spinor Bose gas and the Fermi gas behave like a free spin paramagnet. In this case, the spins are very sensitive to external magnetic fields, with a small field able to polarize all atoms. From equation (14) we obtain

$$
\epsilon(k)=\frac{\hbar^{2} k^{2}}{2 m}-\mu-K_{B} T \ln \left(2 \cosh \frac{H}{2 K_{B} T}\right) .
$$

Under the condition $\gamma \gg 1 / K_{B} T$ and $K_{B} T<T_{d}$, the free energy (12) gives

$$
F(\tau, h) \approx \frac{1}{3} \frac{\hbar^{2}}{2 m} \pi^{2} n^{3}\left[1-\frac{\tau^{2}}{4 \pi^{2}}-\frac{\tau^{4}}{60 \pi^{4}}-\frac{3 \tau}{\pi^{2}} \ln \left(2 \cosh \frac{h}{2 \tau}\right)\right]
$$

where we have set $h=H / T_{d}$.

It follows that in the strong coupling limit $\gamma \rightarrow \infty$ the magnetic properties of the two-component spinor Bose gas are those of $s u(2)$ free spins with a divergent susceptibility $\chi \approx \frac{n}{4 K_{B} T}\left(1-\tanh ^{2}\left(H /\left(2 K_{B} T\right)\right)\right)$ at low temperatures. The magnetization is $m^{z}=\frac{1}{2} n \tanh \left(H /\left(2 K_{B} T\right)\right)$. Figure 1 shows the free spin behaviour in the magnetization as a function of the magnetic field at different temperatures.

The specific heat and entropy follow from equation (36) as

$$
\begin{aligned}
c_{v} & \approx \frac{1}{6} N K_{B} \tau+\frac{N K_{B} \tau^{3}}{15 \pi^{2}}+\frac{N K_{B} h^{2}}{4 \tau^{2}}\left[1-\tanh ^{2}\left(\frac{h}{2 \tau}\right)\right] \\
S & \approx \frac{1}{6} N K_{B} \tau+\frac{N K_{B} \tau^{3}}{45 \pi^{2}}+N K_{B} \ln \left[2 \cosh \left(\frac{h}{2 \tau}\right)\right]-\frac{N K_{B} h}{2 \tau} \tanh \left(\frac{h}{2 \tau}\right) .
\end{aligned}
$$

In the absence of external field $(H=0)$ the specific heat behaves like that of a free Fermi gas. However, the free spins make a contribution $N K_{B} \ln 2$ to the free Fermi entropy. Figure 2 shows a plot of the specific heat in the presence of magnetic field. We see clearly here in the strong coupling limit that the specific heat is sensitive to the external field. We also note that our calculations differ from those in Refs [22, 39] where the spin degrees of freedom were ignored.

\section{B. Ferromagnetic: $1 \ll \gamma<1 / K_{B} T$}

For finite temperatures the solutions of the TBA equations (16) vary from the free spin solutions (21) and (34) $[30,35]$. The spin-spin exchange interactions are enhanced as the interaction strength $\gamma$ decreases from the strong coupling limit. In this section we explore the ferromagnetic behaviour of the spinor Bose gas with finitely strong interaction, i.e., in the regime $1 \ll \gamma<1 / K_{B} T$, or more precisely speaking, $\frac{c}{2 P(T, 0)}<1 / K_{B} T$. In this regime, the known results for the free energy of the ferromagnetic Heisenberg chain at low temperatures obtained by Takahashi and colleagues are applicable to the ferromagnetic state associated with the free energy (15). The result is [36, 51]

$$
f_{X X X}(T, 0) \approx J\left[-1.042\left(\frac{K_{B} T}{J}\right)^{\frac{3}{2}}+\left(\frac{K_{B} T}{J}\right)^{2}-0.9\left(\frac{K_{B} T}{J}\right)^{\frac{5}{2}}\right]
$$




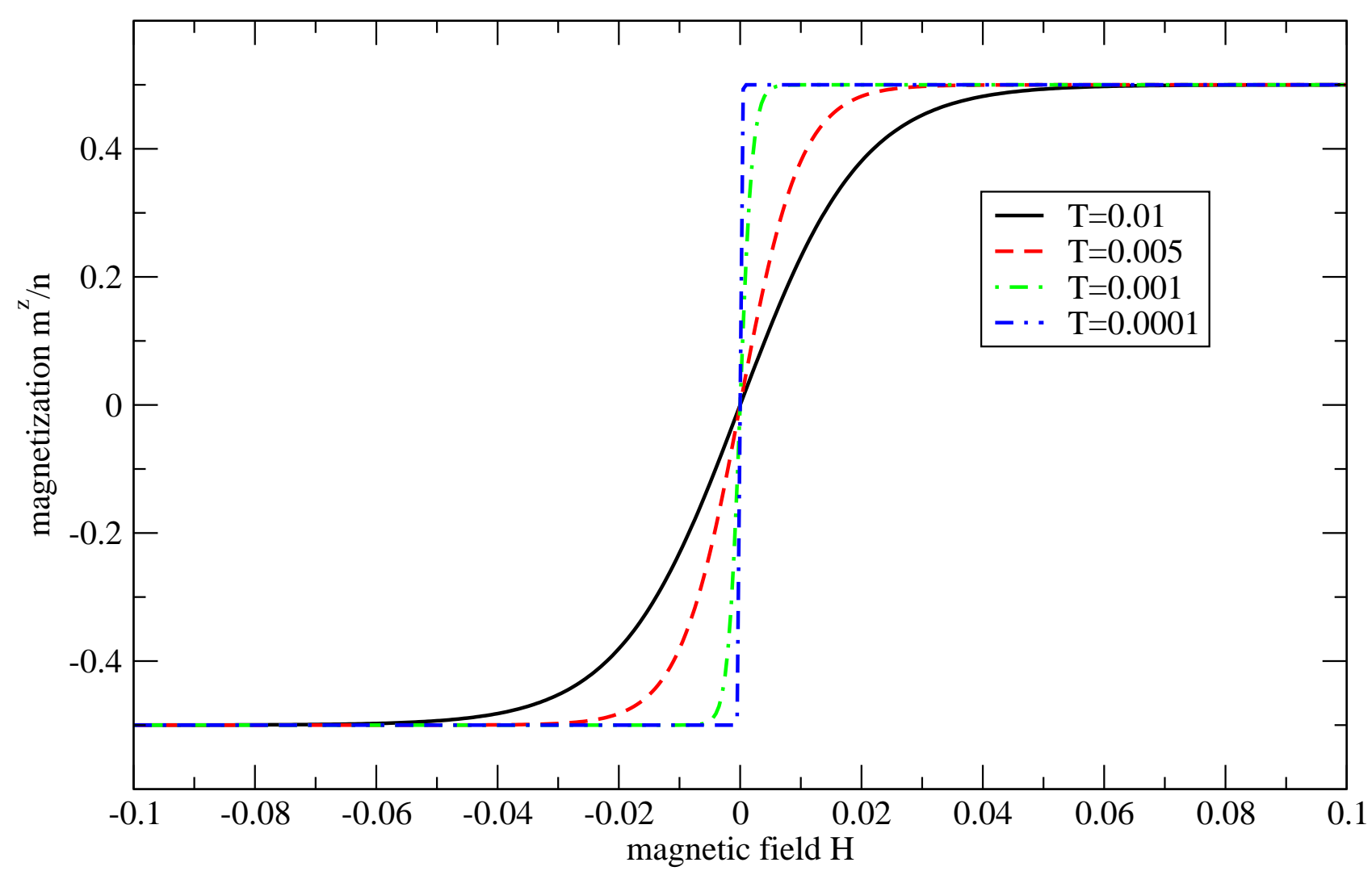

FIG. 1: (color online) Magnetization (normalized by the linear density $n$ ) vs magnetic field $H$ (with Bohr magneton $\mu_{B}=1$ ) for different temperatures (in units of $K_{B}$ ). In the strong coupling limit $\gamma \rightarrow \infty$ two-component spinor bosons exhibit free spin behaviour at low temperatures.

with effective coupling strength $J \approx 2 P(T, 0) / c$. At low temperature, the effective coupling approaches the constant value $J \approx 4 E_{\mathrm{F}} / 3 \gamma$ as a result of the temperature-dependent part in the coupling only making an $O\left(\left(k_{B} T\right)^{3}\right)$ contribution to the free energy of the spinor Bose gas. Schlottmann [35] calculated the leading terms of the specific heat and free energy for the ferromagnetic Heisenberg chain by approximating the infinite set of TBA equations (16). Using Schlottmann's method we find from the TBA equations (16) that the leading order in the free energy is proportional to $\left(K_{B} T\right)^{\frac{3}{2}}$. With the help of Takahashi's result for the ferromagnetic Heisenberg chain (39), we may calculate the thermodynamics of the spinor Bose gas within finitely strong interaction, which we now do.

Substituting $A(T, 0)=\mu+2 P(T, 0) / c-f_{X X X}(T, 0)$ into equation (18) we have

$$
P(T, 0) \approx \frac{1}{\sqrt{\frac{\pi^{2} \hbar^{2}}{2 m}}} \frac{2 \tilde{\mu}^{\frac{3}{2}}}{3}\left[1+\frac{2}{c} \frac{\sqrt{\tilde{\mu}}}{\sqrt{\frac{\pi^{2} \hbar^{2}}{2 m}}}+\frac{\pi^{2}}{8}\left(\frac{K_{B} T}{\tilde{\mu}}\right)^{2}+\frac{\pi^{2}}{6}\left(\frac{K_{B} T}{\tilde{\mu}}\right)^{2} \frac{\sqrt{\tilde{\mu}}}{c \sqrt{\frac{\pi^{2} \hbar^{2}}{2 m}}}\right]
$$

where $\tilde{\mu}=\mu-f_{X X X}(T, 0)$. For the regime $\frac{c}{2 P(T, 0)}<1 / K_{B} T$ calculation of the chemical potential via the relation $\partial P(T, 0) / \partial \mu=n$ gives

$$
\mu \approx \mu_{0}\left[1+\mu_{1}\left(\frac{\gamma K_{B} T}{\mu_{0}}\right)^{\frac{3}{2}}+\mu_{2}\left(\frac{\gamma K_{B} T}{\mu_{0}}\right)^{2}+\mu_{3}\left(\frac{\gamma K_{B} T}{\mu_{0}}\right)^{\frac{5}{2}}+\frac{\pi^{2}}{12}\left(1-\frac{16}{3 \gamma}\right)\left(\frac{K_{B} T}{\mu_{0}}\right)^{2}\right]
$$

where

$$
\mu_{1}=\frac{1.042 \times \sqrt{3}}{4 \gamma}\left(1-\frac{17}{3 \gamma}\right), \quad \mu_{2}=-\frac{3}{2 \gamma}\left(1-\frac{7}{3 \gamma}\right), \quad \mu_{3}=\frac{0.9 \times 21 \sqrt{3}}{16 \gamma}\left(1-\frac{11}{7 \gamma}\right) .
$$

Some algebra gives the free energy $F(\tau, 0)$ and total energy $E(\tau, 0)$ per unit length

$$
F(\tau, 0) \approx E_{0}\left[1-\frac{1.042 \times 3 \sqrt{3}}{2 \gamma \pi^{3}}\left(1+\frac{7}{\gamma}\right)(\gamma \tau)^{\frac{3}{2}}+\frac{9}{4 \gamma \pi^{4}}\left(1+\frac{10}{\gamma}\right)(\gamma \tau)^{2}-\frac{0.9 \times 9 \sqrt{3}}{8 \gamma \pi^{5}}\left(1+\frac{13}{\gamma}\right)(\gamma \tau)^{\frac{5}{2}}\right.
$$




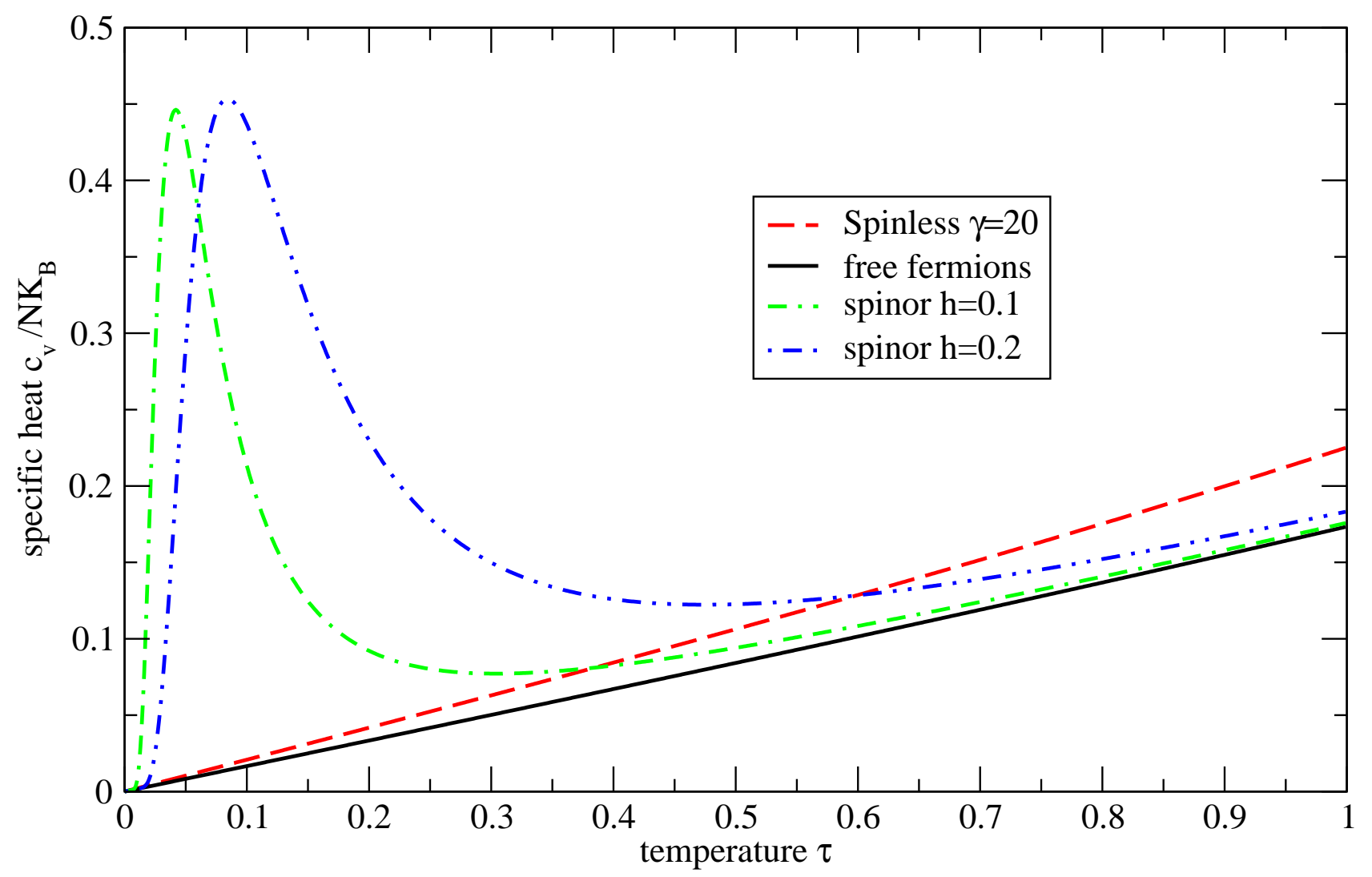

FIG. 2: (color online) Specific heat normalized by $N K_{B}$ vs degenerate temperature $\tau=K_{B} T / T_{d}$. The straight dashed line is the specific heat (27) for the spinless Bose gas with $\gamma=20$. The solid line is the specific heat for the free fermions. The curves show the specific heat (37) of the spinor Bose gas with $\gamma \rightarrow \infty$ for finite field term $h$.

$$
\begin{gathered}
\left.-\frac{\tau^{2}}{4 \pi^{2}}\left(1+\frac{8}{\gamma}\right)\right] \\
E(\tau, 0) \approx E_{0}\left[1+\frac{1.042 \times 3 \sqrt{3}}{4 \gamma \pi^{3}}\left(1+\frac{7}{\gamma}\right)(\gamma \tau)^{\frac{3}{2}}-\frac{9}{4 \gamma \pi^{4}}\left(1+\frac{10}{\gamma}\right)(\gamma \tau)^{2}+\frac{0.9 \times 27 \sqrt{3}}{16 \gamma \pi^{5}}\left(1+\frac{13}{\gamma}\right)(\gamma \tau)^{\frac{5}{2}}\right. \\
\left.+\frac{\tau^{2}}{4 \pi^{2}}\left(1+\frac{8}{\gamma}\right)\right] .
\end{gathered}
$$

We see explicitly that the ferromagnetic behaviour of the spin exchange interaction dominates the thermodynamics of the strongly interacting two-component Bose gas.

It is of interest to note that the leading temperature-dependent term in the energy expressions is $O\left(T^{3 / 2}\right)$ for the spinor Bose gas compared to $O\left(T^{2}\right)$ for the spinless Bose gas (26). In the strong interaction regime $1 \ll \gamma<\mu_{0} / K_{B} T$ this leads to quantitatively different low temperature behaviour compared to the spinless Bose gas. Figure 3 shows the total energy and the free energy in the strong interaction regime as a function of temperature for the spinor Bose gas, the spinless Bose gas and an ideal gas obeying GES. Significantly different characteristics of low temperature behaviour for the spinor Bose gas and the spinless Bose gas are depicted. For the spinless Bose gas with strong coupling, the low temperature thermodynamics is known to coincide with that of ideal particles obeying nonmutual GES with statistics parameter $\alpha \approx 1-2 / \gamma$. Figure 3 hints that the strongly interacting spinor Bose gas might also be equivalent to a gas of ideal particles obeying nonmutual GES. It is an open question as to what the statistics parameter $\alpha$ for the spinor Bose gas might be.

To conclude this section the specific heat and entropy are given by

$$
\frac{c_{v}}{N K_{B}} \approx \frac{1.042 \times 3 \sqrt{3}(\gamma \tau)^{\frac{1}{2}}}{8\left(1-\frac{3}{\gamma}\right) \pi}-\frac{3(\gamma \tau)}{2\left(1-\frac{6}{\gamma}\right) \pi^{2}}+\frac{0.9 \times 45 \sqrt{3}(\gamma \tau)^{\frac{3}{2}}}{32\left(1-\frac{9}{\gamma}\right) \pi^{3}}+\frac{\tau}{6\left(1-\frac{4}{\gamma}\right)}
$$




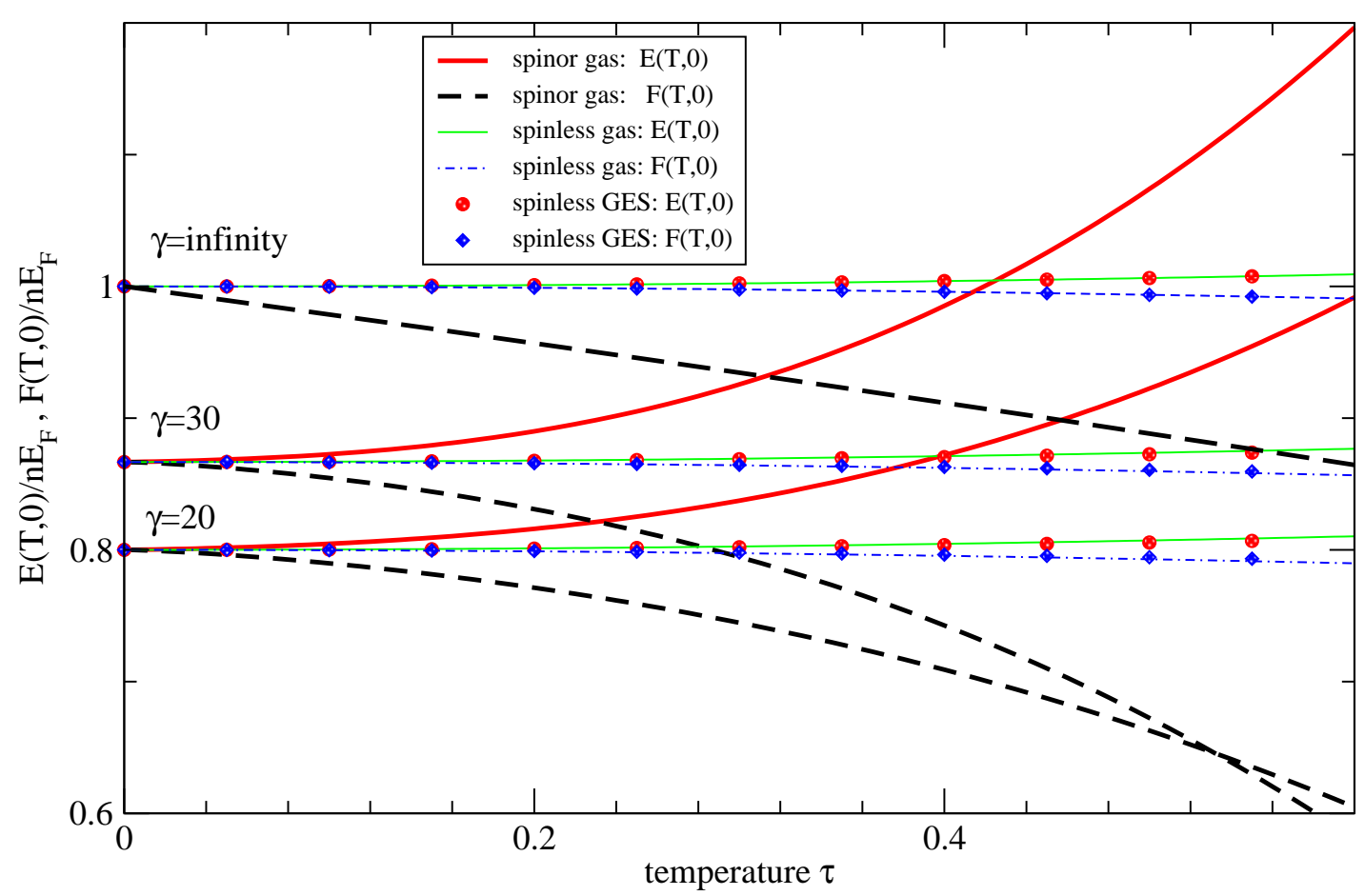

FIG. 3: (color online) Free energy $F(T, 0)$ and total energy $E(T, 0)$ per unit length (normalized by $n E_{\mathrm{F}}=\frac{\hbar^{2}}{2 m} \pi^{2} n^{3}$ ) as a function of the degenerate temperature $\tau=K_{B} T / T_{d}$ for different coupling strengths for both the two-component spinor and spinless 1D Bose gases. The thick solid and dashed lines are the energies (42) and (43) of the spinor Bose gas. The flatter thin solid and dashed lines are the results (26) and (29) for the spinless Bose gas. For further comparison the symbols show the energies (32) and (33) derived for the spinless Bose gas from an ideal gas of particles obeying nonmutual GES [41]. In general the curves reveal the universal characteristics of the energies at low temperatures.

$$
\frac{S}{N K_{B}} \approx \frac{1.042 \times 3 \sqrt{3}(\gamma \tau)^{\frac{1}{2}}}{4\left(1-\frac{3}{\gamma}\right) \pi}-\frac{3(\gamma \tau)}{2\left(1-\frac{6}{\gamma}\right) \pi^{2}}+\frac{0.9 \times 15 \sqrt{3}(\gamma \tau)^{\frac{3}{2}}}{16\left(1-\frac{9}{\gamma}\right) \pi^{3}}+\frac{\tau}{6\left(1-\frac{4}{\gamma}\right)}
$$

which differ significantly from the corresponding spinless Bose gas results (27) and (28) and also from free spin case (37) and (38). Figure 4 shows the specific heat as a function of the temperature. The specific heat exponent $c_{v} \simeq T^{-a}$ indicates that $a=-0.5$ for spinor Bose gas for the regime $1 \ll \gamma<\mu_{0} / K_{B} T$ whereas $a=-1$ for the Lieb-Lininger gas. For $\gamma \gg 1 / K_{B} T$, the spinor Bose gas and the Lieb-Lininger gas both have $a=-1$ for the absence of the external field.

\section{Susceptibility: $1 \ll \gamma<1 / K_{B} T$}

Now we consider the effect of a small external field $(H \ll T)$ within the regime $1 \ll \gamma<1 / K_{B} T$. Here we adapt the known free energy result for the ferromagnetic chain in the presence of an external field [36, 51], namely

$$
f_{X X X}(T, H) \approx f_{X X X}(T, 0)-\frac{H^{2} J}{8\left(K_{B} T\right)^{2}}\left[\frac{1}{6}+0.5826\left(\frac{K_{B} T}{J}\right)^{\frac{1}{2}}+0.678\left(\frac{K_{B} T}{J}\right)\right]
$$

with $f_{X X X}(T, 0)$ as given in (39). Repeating the procedure of the previous section with the free energy (46) gives the result

$$
F(\tau, h) \approx F(\tau, 0)-\frac{E_{0} h^{2}}{\tau^{2}}\left[\frac{1}{12 \gamma}\left(1-\frac{2}{\gamma}\right)+\frac{0.5826 \times \sqrt{3}}{4 \gamma \pi}\left(1-\frac{1}{\gamma}\right)(\gamma \tau)^{\frac{1}{2}}+\frac{0.678 \times 3}{8 \gamma \pi^{2}}\left(1+\frac{4}{\gamma}\right)(\gamma \tau)\right]
$$

with $F(\tau, 0)$ as given in (42). 


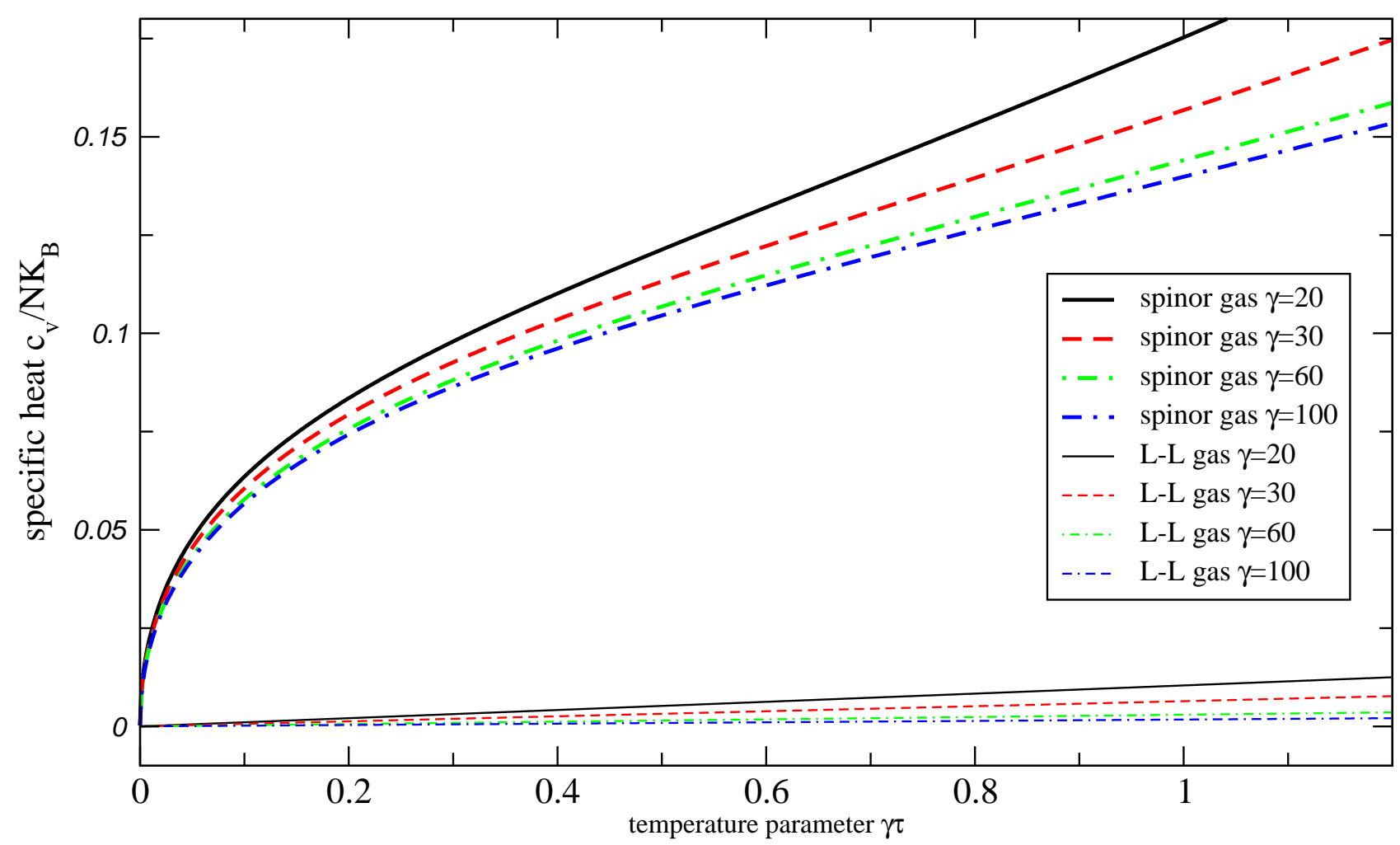

FIG. 4: (color online) Comparison between the specific heat (normalized by $N K_{B}$ ) of the two-component spinor Bose gas and the spinless Bose gas as a function of the temperature parameter $\gamma \tau$ at different coupling strengths. The upper set of curves are obtained from the spinor Bose gas specific heat (44). The lower set of curves follow from the spinless Bose gas result (37). In general the curves highlight the distinction between the temperature-dependent behaviour of the spinor and spinless Bose gases.

For small magnetic field $(h<\tau)$ the susceptibility per unit length

$$
\chi=-\frac{\partial^{2} F(T, H)}{\partial H^{2}} \approx \frac{n}{T_{d}}\left[\frac{\pi^{2}}{18 \gamma \tau^{2}}\left(1-\frac{6}{\gamma}\right)+\frac{0.5826 \times \sqrt{3} \pi}{6 \sqrt{\gamma} \tau^{\frac{3}{2}}}\left(1-\frac{3}{\gamma}\right)+\frac{0.678}{4 \tau}\right]
$$

is indeed greater than that of free spins, for which $\chi \approx n /\left(4 K_{B} T\right)$. This result is also consistent with Eisenberg and Lieb's general argument for polarized spinor bosons [23]. Figure 5 shows the zero-field susceptibility as a function of temperature for different values of the interaction strength. The susceptibility diverges as $\tau \rightarrow 0$. The susceptibility decreases with increasing interaction towards the free spin paramagnetic susceptibility is the lowest curve (solid line). The susceptibility exponent defined by $\chi \sim T^{-b}$ is $b=2$ for the regime $\gamma<\mu_{0} / K_{B} T$ with $b=1$ for the paramagnet.

\section{LOCAL PAIR CORRELATION}

The local pair correlation function for the 1D Bose gas has been determined experimentally in a gas ${ }^{87} \mathrm{Rb}$ atoms as a function of the interaction strength by measuring photoassociation rates [55]. In general local two-particle correlations can be used to study phase coherence behaviour and classify various finite temperature regimes in 1D interacting quantum gases $[56,57]$. In the grand canonical description, two-particle pair correlation are given in terms of the field operator $\Psi$ and the free energy $f(\gamma, T)$ by $[29,56]$

$$
g^{(2)}(0):=\left\langle\Psi^{\dagger} \Psi^{\dagger} \Psi \Psi\right\rangle=\frac{2 m}{\hbar^{2} n^{2}}\left(\frac{\partial f(\gamma, T)}{\partial \gamma}\right)_{n, t} .
$$

At zero temperature the local pair correlation is $g^{(2)} \approx 1$ for the weakly interacting Bose gas. On the other hand, $g^{(2)} \rightarrow 0$ as $\gamma$ increases into the Tonks-Girardeau regime, indicative of free fermionic behaviour. In this regime, the long 


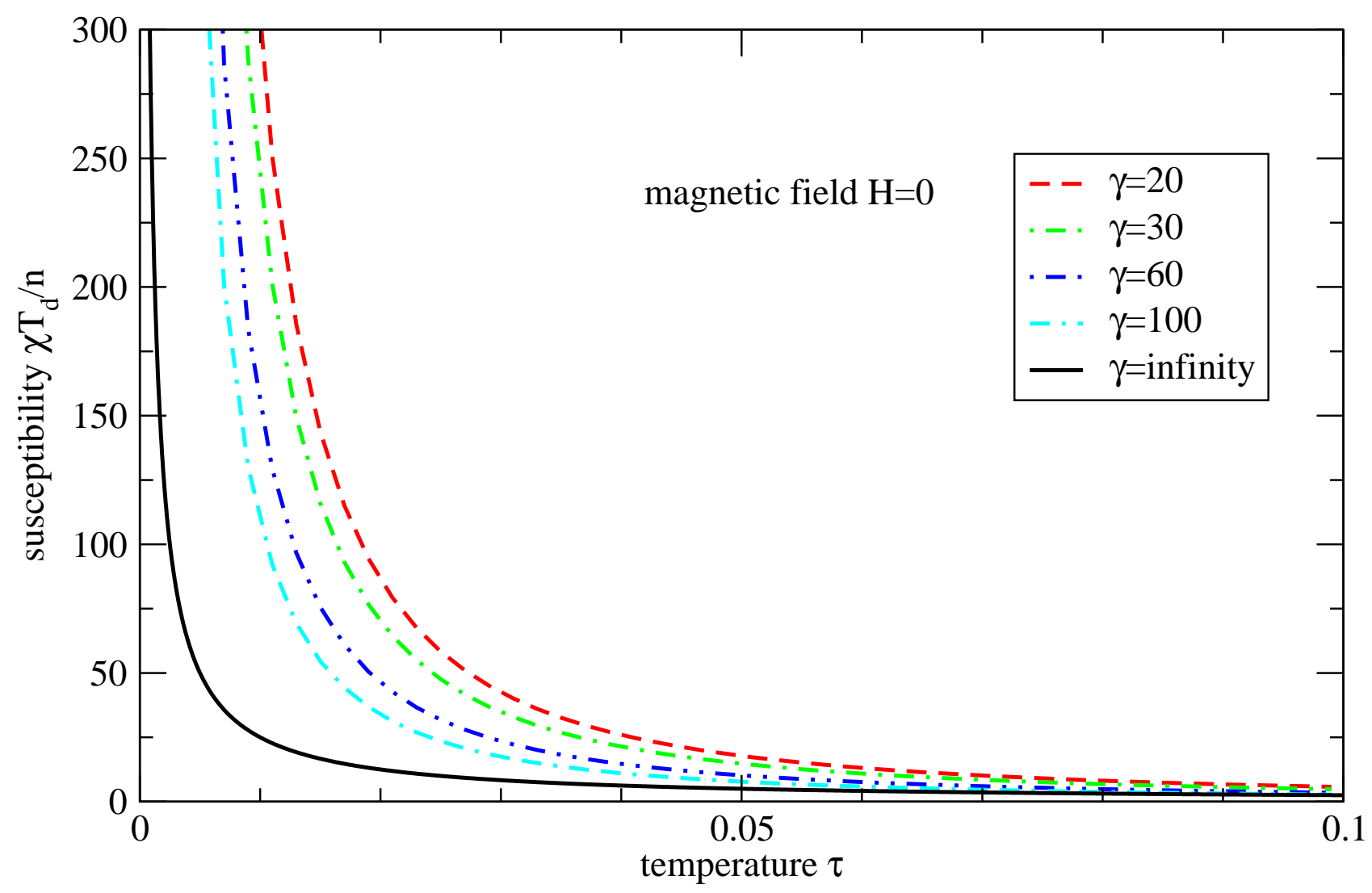

FIG. 5: (color online) Susceptibility (normalized by $n / T_{\mathrm{d}}$ ) as a function of the degenerate temperature $\tau$ for different values of interaction strength $\gamma$. In the strong coupling limit $\gamma \rightarrow \infty$ the two-component spinor bosons behave like a paramagnet with susceptibility $\chi \sim T^{-1}$. For finitely strong interaction, the ferromagnetic susceptibility behaves as $\chi \sim T^{-2}$.

range behaviour is characterized by the one-body correlation function $g^{(1)}(x)=\left\langle\Psi^{\dagger}(x) \Psi(0)\right\rangle \propto 1 / \sqrt{x}$, corresponding to the momentum distribution $n(p) \propto 1 / \sqrt{p}$. Here $p$ is the momentum. More generally, in terms of the Luttinger parameter $K[58], g^{(1)}(x) \propto 1 / x^{1 / 2 K}$ and $n(p) \propto 1 / p^{(1-1 / 2 K)}$. In the weak coupling limit $K \approx \pi / \sqrt{\gamma}$, which leads to a power-law decay in the one point correlation.

The pair correlation function for the spinless Bose gas

$$
g^{(2)}(0) \approx \frac{4 \pi^{2}}{3 \gamma^{2}}\left(1+\frac{\tau^{2}}{4 \pi^{2}}+\frac{\tau^{4}}{20 \pi^{4}}\right)
$$

follows from the TBA result (26), which coincides with the result given in Refs. [56, 57]. It is evident that the dynamical interaction dramatically reduces pair correlation due to decoherence between individual wave functions of colliding particles. On the other hand, increasing temperature slowly enhances local pair correlation. At temperatures $\tau \ll 1$ $\left(T \ll T_{d}\right)$, the local pair correlation approaches free Fermi behaviour as $\gamma \rightarrow \infty$, as was quantitatively demonstrated in the experimental observations of the pair correlation function for a gas of interacting ${ }^{87} \mathrm{Rb}$ atoms confined to $1 \mathrm{D}$ $[55]$.

For the two-component spinor Bose gas considered here, the ferromagnetic spin-spin exchange interaction results in a different temperature-dependent pair correlation function. For this model, the local pair correlation function

$$
g^{(2)}(0) \approx \frac{4 \pi^{2}}{3 \gamma^{2}}\left[1-\frac{1.042 \times 3 \sqrt{3}(\gamma \tau)^{\frac{3}{2}}}{16 \pi^{3}}\left(1-\frac{3}{\gamma}\right)+\frac{9(\gamma \tau)^{2}}{16 \pi^{4}}-\frac{0.9 \times 27 \sqrt{3}(\gamma \tau)^{\frac{5}{2}}}{64 \pi^{5}}\left(1+\frac{3}{\gamma}\right)+\frac{\tau^{2}}{4 \pi^{2}}\right]
$$

follows from equation (42) in the regime $\mu_{0} / K_{B} T>\gamma \gg 1$. We see for the spinor Bose gas the local pair correlation again quickly decays with respect to the dynamical interaction $\gamma$. Figure (6) shows the local pair correlation for the spinless and the spinor Bose gases as a function of the interaction strength at different temperatures. In contrast to the spinless Bose gas, where the temperature enhances local pair correlation due to thermal fluctuations, for the 

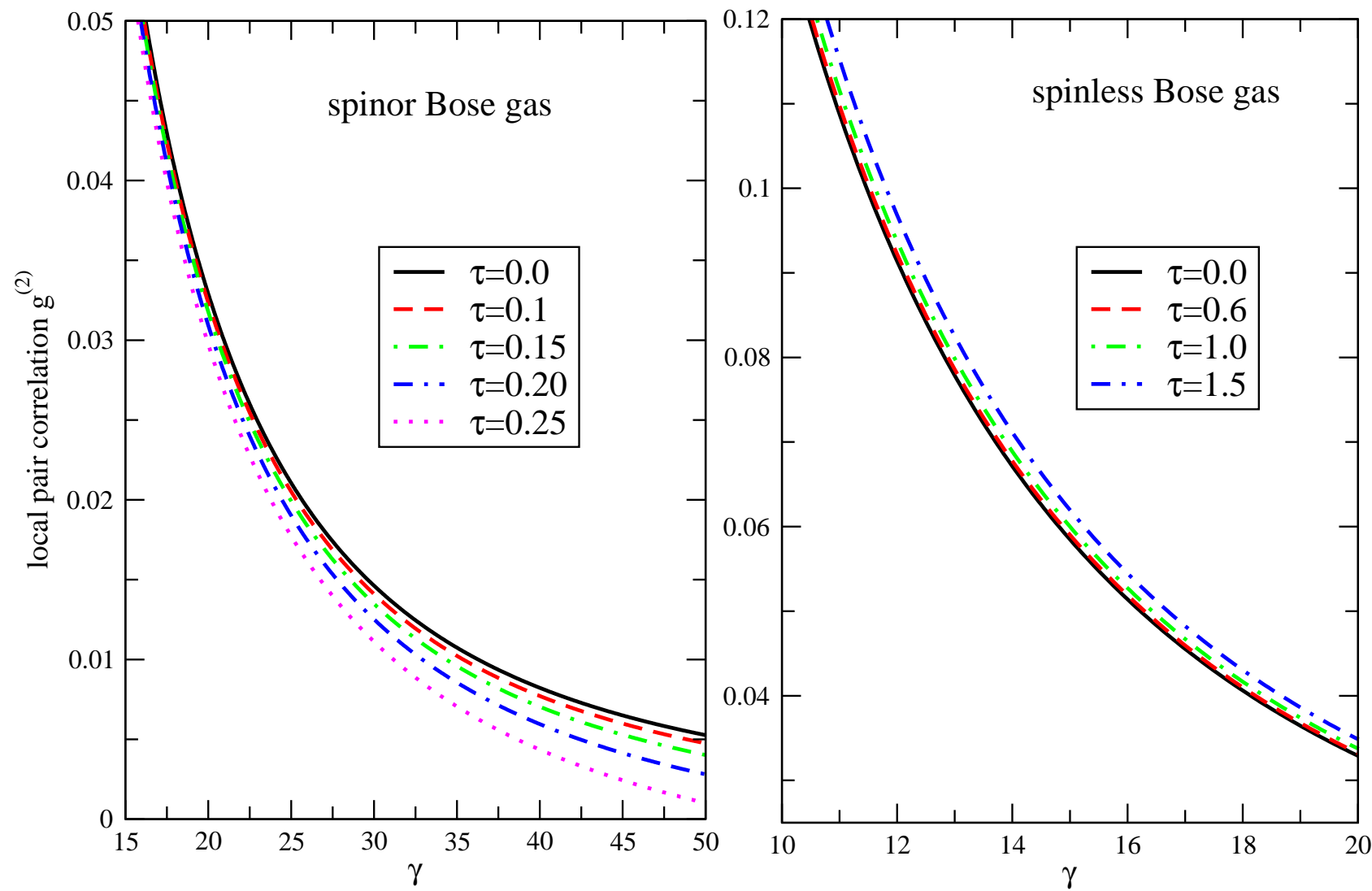

FIG. 6: (color online) Local pair correlation $g^{(2)}$ as a function of interaction strength $\gamma$ at different temperatures. For the spinless Bose gas, the pair correlation function increases with increasing temperature. In contrast, for the two-component spinor Bose gas the pair correlation function decreases with increasing temperature as a result of the temperature induced spin-spin ferromagnetic exchange interaction.

spinor Bose gas the local pair correlation decreases with increasing temperature due to the ferromagnetic spin-spin exchange interaction.

\section{CONCLUSION}

In this paper we have studied the thermodynamics of the integrable 1D two-component Bose gas via the thermodynamic Bethe ansatz. Analytic low temperature results were obtained for the free energy, total energy, specific heat, entropy, pressure, susceptibility and pair correlation function in the strongly interacting regimes $\gamma \gg 1 / K_{B} T$ and $1 \ll \gamma<\mu_{0} / K_{B} T$. Where appropriate, comparison was made with corresponding thermodynamic properties of the integrable 1D spinless Bose gas. Our key finding is that the temperature induced ferromagnetic spin-spin exchange interaction triggers a number of novel quantum effects in the thermodynamic properties of the spinor Bose gas at low temperatures. In the regime $1 \ll \gamma<\mu_{0} / K_{B} T$ the specific heat exponent for the spinor Bose gas following from $c_{v} \sim T^{1 / 2}$ is different to that of the spinless Bose gas for which $c_{v} \sim T$. In this regime, the susceptibility exponent is given by $\chi \sim T^{-2}$ which exceeds that of free spin paramagnet for which $\chi \sim T^{-1}$. In contrast to the spinless Bose gas, where the pair correlation function increases with increasing temperature, the two-component spinor Bose gas pair correlation function decreases with increasing temperature as a result of the temperature induced spin-spin ferromagnetic exchange interaction.

In general these exact results should be relevant to understanding ferromagnetic behaviour and spin effects in twocomponent spinor Bose gases of cold atoms, for which the interaction strength can in principle be tuned. However, the introduction of precise thermometry into these systems to measure universal temperature dependent effects provides a number of challenges. However, this is a worthwhile goal. As we have seen, for strong coupling the characteristics of 
the thermodynamics of the spinor Bose gas at low temperatures are described by an effective ferromagnetic Heisenberg spin chain. There is a remarkable three-way correspondence $[53,54]$ between the ferromagnetic Heisenberg chain, the limit of weakly coupled planar $\mathcal{N}=4$ supersymmetric Yang-Mills theory and the limit of free strings on $\operatorname{AdS}_{5} \times$ $S^{5}$. This triality between guage theory, string theory and the thermodynamics of the ferromagnetic Heisenberg chain has recently been used to calculate the Hagedorn temperature of the string theory in agreement with the Hagedorn/deconfinement temperature calculated on the guage theory side [54]. It now appears that we can add a further connection with the thermodynamics of the strongly interacting two component spinor Bose gas.

\section{Acknowledgments}

This work is supported by the Discovery and Linkage International programs of the Australian Research Council through grants DP0342561, DP0663773 and LX0455823. It is also in part supported by Grant-in-Aid for the Scientific Research (B) No. 18340112 from the Ministry of Education, Culture, Sports, Science and Technology, Japan. The authors thank Michael Bortz, Fabian Essler, Andreas Klümper, Masahiro Shiroishi and David Weiss for helpful discussions. M. T. thanks the Department of Theoretical Physics for hospitality during the initial part of this work. X.-W. G. and M. T. B. thank the Institute of Solid State Physics, University of Tokyo and Institut Henri PoincareCentre Emile Borel for hospitality and support during the final stages.

[1] M. Lewenstein, A. Sanpera, V. Ahufinger, B. Damski, A. Sen and U. Sen, Adv. Phys. 56, 243 (2007).

[2] R. Grimm, arXiv:cond-mat/0703091.

[3] S. Giorgini, L. P. Pitaevskii and S. Stringari, arXiv:0706.3360.

[4] H. Moritz, T. Stöferle, M. Köhl and T. Esslinger, Phys. Rev. Lett. 91, 250402 (2003).

[5] B. L. Tolra, K. M. O'Hara, J. H. Huckans, W. D. Phillips, S. L. Rolston and J. V. Porto, Phys. Rev. Lett. 92, 190401 (2004).

[6] B. Paredes, A. Widera, V. Murg, O. Mandel, S. Folling, I. Cirac, G. V. Shlyapnikov, T. W. Hansch and I. Bloch, Nature 429, 277 (2004).

[7] T. Kinoshita, T. Wenger and D. S. Weiss, Science 305, 1125 (2004).

[8] L. Pollet, S. M. A. Rombouts and P. J. H. Denteneer, Phys. Rev. Lett. 93, 210401 (2004).

[9] E. H. Lieb and W. Liniger, Phys. Rev. 130, 1605 (1963).

[10] J. B. McGuire, J. Math. Phys. 5, 622 (1964).

[11] H. J. Lewandowski, D. M. Harber, D. L. Whitaker and E. A. Cornell, Phys. Rev. Lett. 88, 070403 (2002).

[12] J. M. McGuirk, H. J. Lewandowski, D. M. Harber, T. Nikuni, J. E. Williams and E. A. Cornell, Phys. Rev. Lett. 89, $090402(2002)$.

[13] J. M. McGuirk, D. M. Harber, H. J. Lewandowski and E. A. Cornell Phys. Rev. Lett. 91, 150402 (2003).

[14] T.-L. Ho, Phys. Rev. Lett. 81, 742 (1998); T.-L. Ho and S. K. Yip, Phys. Rev. Lett. 84, 4031 (2000).

[15] K. Yang and Y.-Q. Li, Int. J. Mod. Phys. B 17, 1027 (2003).

[16] T. Ohmi and K. Machida, J. Phys. Soc. Jpn 67, 1882 (1998).

[17] S. Ashhab, J. Low Temp. Phys. 140, 51 (2005).

[18] T. Nikuni and J. E. Williams, J. Low. Temp. Phys. 133, 323 (2003).

[19] Y. Kawaguchi, H. Saito and M. Ueda, Phys. Rev. Lett. 98, 110406 (2007).

[20] M. Erhard, H. Schmaljohann, J. Kronjäger, K. Bongs and K. Sengstock, Phys. Rev. A 69, 032705 (2004); A. Widera, O. Mandel, M. Greiner, S. Kreim, T. W. Hänsch and I. Bloch, Phys. Rev. Lett. 92, 160406 (2004).

[21] B. Sutherland, Phys. Rev. Lett. 20, 98 (1968).

[22] Y.-Q. Li, S.-J. Gu, Z.-J. Ying and U. Eckern, Europhys. Lett. 61, 368 (2003).

[23] E. Eisenberg and E. H. Lieb, Phys. Rev. Lett. 89, 220403 (2002).

[24] J. N. Fuchs, D. M. Gangardt, T. Keilmann and G. V. Shlyapnikov, Phys. Rev. Lett. 95, 150402 (2005).

[25] M. T. Batchelor, M. Bortz, X.-W. Guan and N. Oelkers, J. Stat. Mech. P03016 (2006).

[26] M. B. Zvonarev, V. V. Cheianov and T. Giamarchi, arXiv:0708.3638.

[27] F. Deuretzbacher, et al, arXiv: 0708.3039.

[28] A. Kleine, C. Kollath, I. P. McCulloch, T. Giamarchi and U. Schollwöck, arXiv:0706.0709.

[29] V. E. Korepin, A. G. Izergin and N. M. Bogoliubov, Quantum Inverse Scattering Method and Correlation Functions (Cambridge University Press, Cambridge, 1993).

[30] M. Takahashi, Thermodynamics of One-Dimensional Solvable Models (Cambridge University Press, Cambridge, 1999).

[31] P. Schlottmann, Int. J. Mod. Phys. B 11, 355 (1997).

[32] F. H. L. Essler, H. Frahm, F. Göhmann, A. Klümper, V. E. Korepin, The One-Dimensional Hubbard Model (Cambridge University Press, Cambridge, 2005).

[33] A. M. Tsvelick and P. B. Wiegmann, Adv. Phys. 32, 453 (1983).

[34] C. N. Yang and C. P. Yang, J. Math. Phys. 10, 1115 (1969). 
[35] P. Schlottmann, Phys. Rev. Lett. 54, 2131 (1985).

[36] M. Takahashi and M. Yamada, J. Phys. Soc. Japan, 54, 2808 (1985); M. Takahashi, Phys. Rev. Lett. 58, 168 (1987).

[37] M. Olshanii, Phys. Rev. Lett. 81, 938 (1998).

[38] M. Takahashi, Prog. Theor. Phys. 46, 401 (1971).

[39] S.-J. Gu, Y.-Q. Li, Z.-J. Ying and X.-A. Zhao, Int. J. Mod. Phys. B 16, 2137 (2002).

[40] M. Toda and N. Saito, Statistical Physics I (Springer-Verlag, Heidelberg, 1992).

[41] M. T. Batchelor and X.-W. Guan, Phys. Rev. B 74, 195121 (2006); Laser Phys. Lett. 4 (2007) 77.

[42] I. Affleck, Phys. Rev. Lett. 56, 746 (1986).

[43] H. W. J. Blöte, J. L. Cardy and M. P. Nightingale, Phys. Rev. Lett. 56, 742 (1986).

[44] M. Bortz, J. Phys. A 40, 1 (2007).

[45] M. T. Batchelor, X.-W. Guan and N. Oelkers, Phys. Rev. Lett. 96, 210402 (2006).

[46] F. D. M. Haldane, Phys. Rev. Lett. 67, 937 (1991).

[47] Y.-S. Wu, Phys. Rev. Lett. 73, 922 (1994); D. Bernard and Y.-S. Wu, arXiv:cond-mat/9404025.

[48] S. B. Isakov, Phys. Rev. Lett. 73, 2150 (1994).

[49] G. A. Baker Jr., G. S. Rushbrooke and H. E. Gilbert: Phys. Rev. A 135, 1272 (1964).

[50] J. C. Bonner and M. E. Fisher, Phys. Rev. A 135, 640 (1964).

[51] M. Yamada and M. Takahashi, J. Phys. Soc. Japan 55 , 2024 (1986).

[52] S. J. Blundell and F. L. Pratt, J. Phys.: Condens. Matter 16, R771 (2004).

[53] J. A. Minahan, J. Phys. A. 39, 12657 (2006).

[54] T. Harmark and M. Orselli, Phys. Rev. D 74, 126009 (2006); T. Harmark, K. R. Kristjansson and M. Orselli, JHEP 02 (2007) 085.

[55] T. Kinoshita, T. Wenger and D. S. Weiss, Phys. Rev. Lett. 95, 190406 (2005).

[56] D. M. Gangardt and G. V. Shlyapnikov, Phys. Rev. Lett. 90, 010401 (2003); K. V. Kheruntsyan, D. M. Gangardt, P. D. Drummond and G. V. Shlyapnikov, Phys. Rev. Lett. 91, 040403 (2003).

[57] M. A. Cazalilla, Phys. Rev. A 67, 053606 (2003).

[58] M. A. Cazalilla, J. Phys. B 37, S1 (2004). 